\title{
Review of the Direct Laser Synthesis of Functionalized Graphene and its Application in Sensor Technology
}

\author{
Received August 7, 2019; revised September 17, 2019; accepted September 18, 2019
}

\author{
Seok-Ki Hyeong ${ }^{\mathrm{a}, \mathrm{c}}$, Kwang-Hun Choi ${ }^{\mathrm{b}, c}$, Seoung-Woong Park ${ }^{c}$, Sukang Bae ${ }^{c}$, Min Park ${ }^{\mathrm{d}}$, Seongwoo Ryu ${ }^{\mathrm{b}, *}$, \\ Jae-Hyun Lee ${ }^{\mathrm{a}, *}$, and Seoung-Ki Lee ${ }^{\mathrm{c}, *}$ \\ aDepartment of Energy Systems Research and Department of Materials Science and Engineering, Ajou University, Suwon 16499, Republic \\ of Korea \\ ${ }^{b}$ Department of Polymer Engineering, College of Engineering, Suwon University, Suwon 18323, Republic of Korea \\ ${ }^{c}$ Functional Composite Materials Research Center, Institute of Advanced Composite Materials, Korea Institute of Science and Technology \\ (KIST), Jeollabuk-do 55324, Republic of Korea

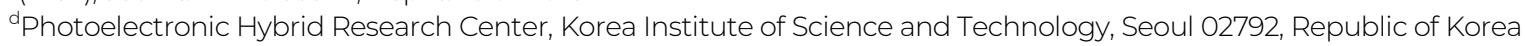

*Corresponding author E-mail: ryu@suwon.ac.kr, jaehyunlee@ajou.ac.kr, sklee@kist.re.kr

\begin{abstract}
Since the breakthrough in fabricating graphene by mechanical exfoliation in 2004, numerous methods have been developed to synthesize high-quality graphene materials on a large scale, including chemical exfoliation, thermolysis, and chemical vapor deposition. Recently, laser thermal treatments have emerged as facile methods for the direct synthesis of functionalized graphene materials, which show potential for use in a wide range of applications. The graphene materials produced by laser-based syntheses are classified by the fabrication method as either laserreduced graphene or laser-induced graphene (LIG). The former is obtained through the chemical reduction of graphene oxide, while the latter utilizes the carbonization of a polymer precursor. In this review, we summarize the mechanisms of laser-assisted graphene syntheses, the structural and chemical functionalization of laser-scribed graphene, and various practical demonstrations of graphene-based materials in the field of mechanical and electrochemical sensors.
\end{abstract}

Keywords: Laser ablation, Graphene, Functionalization, Electromechanical sensor, Electrochemical sensor

\section{Introduction}

With the advent of the Internet of Things (IoT), the importance of sensors that can communicate data by digitizing analog signals in real time has been increasing. Sensor technology is widely used in diverse areas. Two key applications of next-generation sensors are in healthcare, in which the signals produced by the human body are monitored [1]; and in safety inspections, whereby hazardous materials and environmental contaminants are detected, including nitrogen oxide $\left(\mathrm{NO}_{x}\right)$, sulfur oxide $\left(\mathrm{SO}_{x}\right)$, and carbon monoxide $(\mathrm{CO})$ [2]. The latest studies on such next-generation sensors have focused on the design and use of functional materials that offer superior selectivity as well as high sensitivity. In particular, nanostructured materials such as nanoparticles (NPs), nanowires, and nanotubes have attracted considerable attention as active materials [3,4]. Remarkable progress has been made in the field of nanocarbon-based sensor systems, which have a large surface area $[5,6]$, outstanding selectivity and sensitivity $[7,8]$, and more advanced properties than conventional bulk materials. Graphene offers several unique advantages based on its crystal structure, leading to its wide recognition as a suitable material for the development of ultrasensitive sensors. Graphene specimens can detect an external environment through the whole surface; therefore, they have a considerable sensing area per unit volume, which maximizes the sensing effect $[6,9]$. In addition, the mass of the charge carriers in graphene changes drastically depending on the chemical absorbate, allowing superior selectivity [9]. Graphene sensors can also detect fine mechanical deformation owing to their piezoresistive properties [10].

The fabrication of graphene materials has been achieved through mechanical/chemical exfoliation [11], chemical vapor deposition [12], and epitaxial growth [13]. However, these methods require precise synthesis conditions, high temperatures, and complex post-treatments such as transferring or patterning, making them cost-intensive and time-consuming. Recently, a facile method for the synthesis of selectively patterned graphene has been reported by laser scribing [14,15], which largely resolves the above-mentioned drawbacks. Laser-based syntheses allow the rapid realization of graphene within minutes, with good control over the structure and functional groups. As such, laserbased syntheses have been actively studied in various areas. This review paper summarizes recent research trends regarding the synthesis of graphene using laser ablation methods, and the application of the resultant devices as sensors. Depending on the synthesis method and mechanism, such graphene is classified as either laser-reduced graphene (LRG) or laser-induced graphene (LIG). Herein, we first discuss the synthesis of LRG from the reduction of graphene oxide (GO), and its functionalization. Subsequently, we summarize the synthesis and functionalization of LIG from the direct carbonization of a polymer substrate. Finally, we explore the application of these materials as different types of mechanical and electrochemical sensors. 


\section{Laser-based direct synthesis of graphene}

\section{1) Laser-reduced graphene (LRG)}

Synthesis of LRG from graphene oxide (GO)

LRG is synthesized via the reduction of GO by heat treatment through laser energy. The first LRG synthesis method was discovered by Zhang et al. [14], who synthesized reduced graphene oxide (rGO) from a pure GO film by irradiating it with a femtosecond laser [Fig. 1(a)]. Since then, LRG has been synthesized with various laser sources, including nanosecond [16], Nd:YAG [17], $\mathrm{KrF}$ [18], $\mathrm{CO}_{2}$ [19], infrared [20], and fiber lasers [21]. LRG patterns can be directly formed on a substrate by moving the laser over target areas [22], and both vertical rGO-GO-rGO sandwich structures and horizontal structures with micrometer-scale in-plane features can be fabricated [Fig. 1(b)]. Figure 1(c) shows a generalized process for synthesizing LRG on a GOcoated flexible plastic substrate. LRG synthesis is superior to that of conventional graphene, as it transpires under ambient conditions, and intentional patterns can be formed in a facile manner [Fig. 1(d)] without the need for complex transfer, photolithography, or etching processes [23,24]. Strong et al. [22] demonstrated the potential of LRG for application in stretchable electronics by transferring a LRG electrode structure onto a polydimethylsiloxane (PDMS) substrate, as shown in Fig. 1(e).

The mechanism of LRG synthesis is thought to involve both photochemical and photothermal processes, depending on the wavelength of the irradiating laser. The reduction of GO to rGO reportedly occurs via photochemical processes at wavelengths shorter than 390 $\mathrm{nm}(3.2 \mathrm{eV})$ [25], and via photothermal processes at wavelengths longer than $390 \mathrm{~nm}$. In the photochemical process [26], the contamination

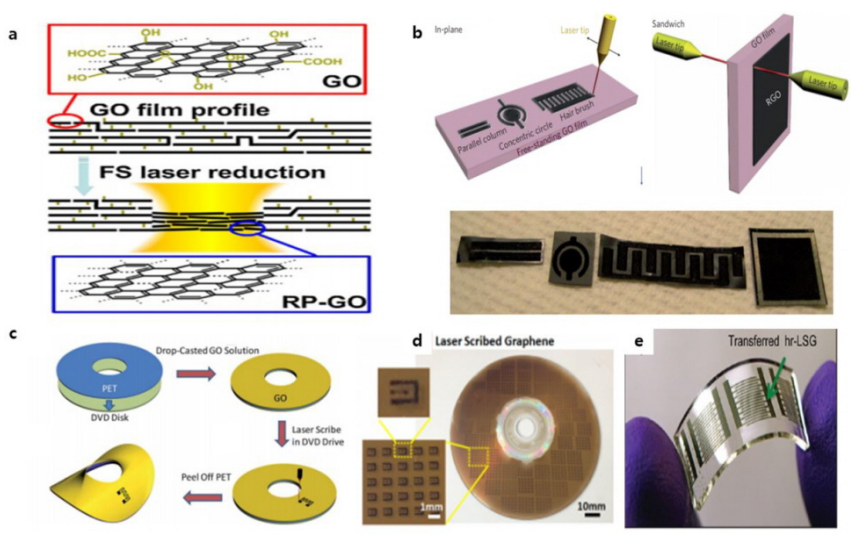

Figure 1. (Color online) (a) Illustration showing RP-GO reduced by a femtosecond (FS) laser from GO. Laser ablation removes oxygen contamination groups, resulting in rGO. (Reprinted with permission from [14] (Y. Zhang et al., Nano Today 5, 15 (2010)), ( ) 2019, Elsevier). (b) Schematics of selective patterning using laser irradiation and photographs of the resultant structures. Hydrated GO films were irradiated to form rGO-GO-rGO devices with in-plane and sandwich geometries. (Reprinted with permission from [19] (W. Gao et al., Nat. Nanotechnol. 6, 496 (2011)), ( 2019 , Nature Publishing Group). (c) Schematic showing the fabrication process of laser-scribed graphene (LSG). (Reprinted with permission from [23] (H. Tian et al., Nanoscale 6, 699 (2014)), ( 2 2019, Royal Society of Chemistry). (d) Photograph of wafer-scale patterned LSG. (Reprinted with permission from [24] (H. Tian et al., Sci. Rep. 4, 3598 (2014)), ( 2019, Springer Nature). (e) Photograph of flexible LRG electrodes on a polydimethylsiloxane (PDMS) substrate. (Reprinted with permission from [22] (V. Strong et al., ACS Nano 6, 1395 (2012)), ( 2019, American Chemical Society). groups of GO are removed owing to the heat produced by the laser, causing the carbon atoms to rearrange to form $\mathrm{sp}^{2}$ bonds. Similarly, in the photothermal process, the bonds of oxygen-containing groups $(-\mathrm{OH},-\mathrm{COOH})$ are broken and removed due to the high local heat (> $2500{ }^{\circ} \mathrm{C}$ ). Figure 2(a) shows optical and scanning electron microscopy (SEM) images of LRG synthesized through laser treatment. The gray and black parts indicate LRG and GO, respectively. The graphene can be selectively synthesized in a single direction according to the scribing direction of the laser [27]. In addition, the synthesized LRG has a layered structure, which is typical for rGO, with parallel vacancies observed along the cross-section. Figure 2(b) shows the C1s spectra of LRG obtained using X-ray photoelectron (XP) spectroscopy. The three main peaks indicate $\mathrm{C}-\mathrm{C}$ (non-oxygenated ring carbon, $284.6 \mathrm{eV}$ ), C-O (hydroxyl and epoxy carbon, $286.6 \mathrm{eV}$ ), and $\mathrm{C}=\mathrm{O}$ (carbonyl, $288.5 \mathrm{eV}$ ). The results show that the oxygen groups dramatically decrease after laser irradiation. As shown in Fig. 2(c), the proportion of $\mathrm{C}-\mathrm{O}$ and $\mathrm{C}=\mathrm{O}$ decreases as the laser power increases, whereas the proportion of $\mathrm{C}-\mathrm{C}$ increases.

\section{Functionalization}

The electrical and mechanical properties of LRG can be improved by doping with metal NPs, metal oxide, and chemical functional groups. For example, Strong et al. [22] grew Pt NPs with a size of 10$150 \mathrm{~nm}$ on LRG via electrodeposition [Fig. 3(a)], leading to improved performance. Pt-doped LRG has the potential to be applied in devices in the electrochemical field. In another approach, Chen et al. [28] developed an in situ synthesis method to combine LRG with $\mathrm{MnO}_{2}$ $\mathrm{NPs}$ by irradiating a graphene- $\mathrm{MnO}_{2}$ composite. This approach prevents agglomeration of the graphene sheet during laser irradiation, resulting in graphene with an improved specific surface area. Consequently, a graphene composite with improved electrochemical performance can be fabricated using a simple and inexpensive method compared with conventional methods [Fig. 3(b)].

Another method of improving the performance of LRGs involves chemical functionalization. The fluorination of graphene can be achieved by irradiating a fluoropolymer-coated graphene substrate with a laser [Fig. 3(c)]. Fluorine radicals are locally generated upon laser scribing,
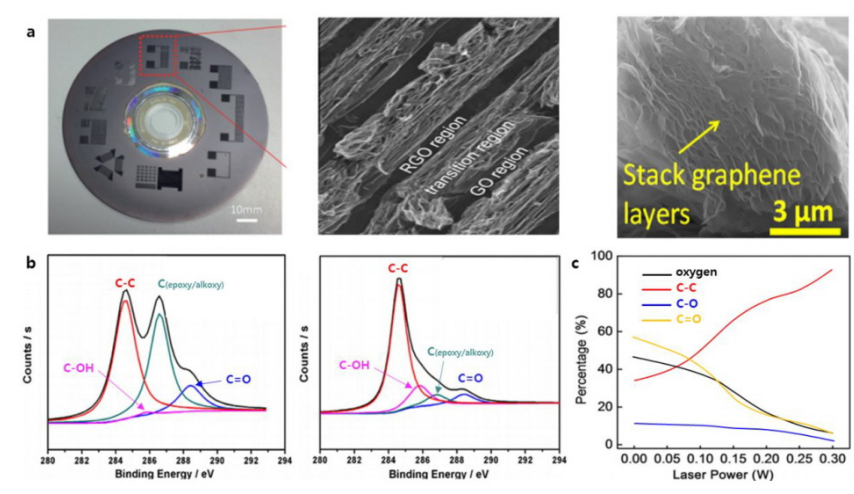

Figure 2. (Color online) (a) Photographs and SEM images of LRG. In the SEM image (left), dark region $\mathrm{rGO}$ and bright region $\mathrm{rGO}$ are shown. The high density of LRG can be seen in the cross-sectional view (right). (Reprinted with permission from [24] (H. Tian et al., Sci. Rep. 4, 3598 (2014)), @ 2019, Springer Nature; and [23] (H. Tian et al., Nanoscale 6, 699 (2014)), (c 2019, Royal Society of Chemistry). (b) XP C1s spectra of GO and rGO before (left) and after (right) laser irradiation. (c) Dependence of the laser power on the atomic percentage of $\mathrm{C}-\mathrm{C}, \mathrm{C}-\mathrm{O}$, and $\mathrm{C}=\mathrm{O}$ in rGO films. 

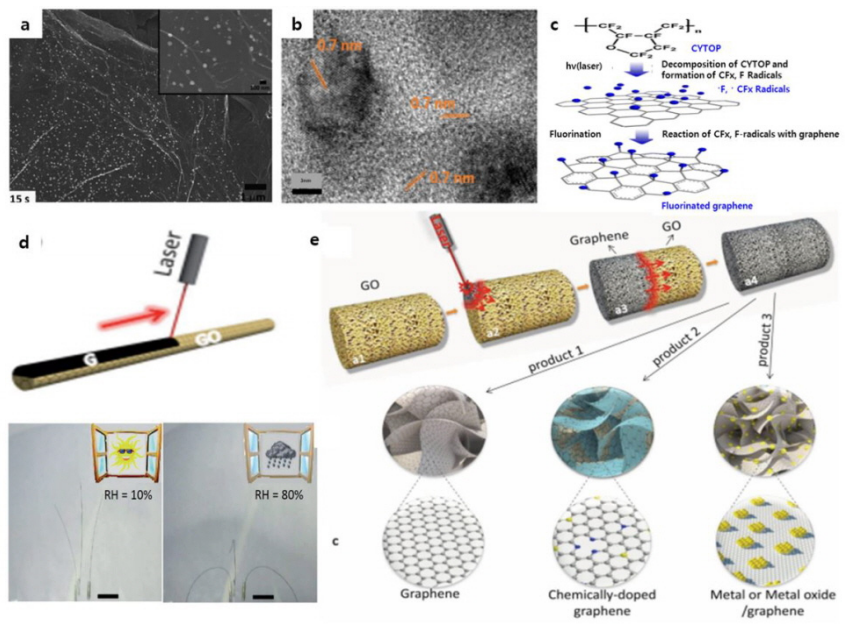

Figure 3. (Color online) (a) SEM image of electrodeposited Pt NPs on LSG. The white particles are Pt NPs. (Reprinted with permission from [22] (V. Strong et al., ACS Nano 6, 1395 (2012)), @ 2019, American Chemical Society). (b) TEM image of MnO on LSG. (Reprinted with permission from [28] (Y. Chen et al., J. Mater. Sci.: Mater. Electron. 27, 2564 (2016)), @ 2019, Nature Publishing Group). (c) Schematic of the synthesis of fluorinated LSG from CYTOP by laser irradiation. (Reprinted with permission from [29] (W. H. Lee et al., Nano Lett. 12, 2374 (2012)), @ 2019, American Chemical Society). (d) LRG fiber. G-graphene (dark region); GO-graphene oxide (brown region). Three-wire moisture sensors made of graphene/GO fibers on a sunny (left) and rainy (right) day. (Reprinted with permission from [30] (H. Cheng et al., Angew. Chem. Int. Ed. 52, 10482 (2013)), @ 2019, John Wiley and Sons). (e) Schematic of the preparation of bulk graphene. (Reprinted with permission from [31] (H. Cheng et al., Adv. Mater. 28, 3305 (2016)), @ 2019, John Wiley and Sons).

which induces $\mathrm{C}-\mathrm{F}$ bonding on the graphene sheets. The kinetics of $\mathrm{C}$ -F bond formation are affected by the laser power and fluoropolymer thickness [29]. Notably, selective synthesis using this method facilitates the structural functionalization of graphene. Cheng et al. [30] partially reduced a GO fiber by irradiating it with an argon laser $(\lambda=458 \mathrm{~nm})$, thereby achieving an $\mathrm{rGO} / \mathrm{GO}$ fiber with anisotropic properties in the longitudinal direction [Fig. 3(d)]. Owing to its "shape memory," the partially reduced carbon composite fiber could be applied as a smart textile that changes shape depending on the humidity. Figure 3(e) illustrates the results of the chemical and structural functionalization of bulk LRG. By laser irradiation of a graphene aerogel that was impregnated with a dopant such as a metal or metal oxide, a functional graphene composite could be quickly fabricated. The fabricated functional graphene material exhibited a high energy conversion efficiency and high performance as an energy storage device owing to its high electric conductivity and large surface area [31].

\section{2) Laser-induced graphene (LIG)}

\section{Synthesis of LIG from polymer precursors}

While LRG technology facilitates the selective synthesis of graphene, the preparation is lengthy on account of the formation of the GO film that is used as source material. In contrast, LIG is synthesized directly from a polymer precursor using functionalization, reducing the overall processing time. Figure 4(a) shows a representative process for the synthesis of LIG. Carbonization of the source polymer occurs owing to local heating upon laser irradiation, allowing graphene to be formed selectively. During the carbonization process, the oxygen and nitrogen groups in the substrate are decomposed; simultaneously, the carbon atoms in $\mathrm{sp}^{3}$ bonds reform as $\mathrm{sp}^{2}$ bonds [15]. Typical
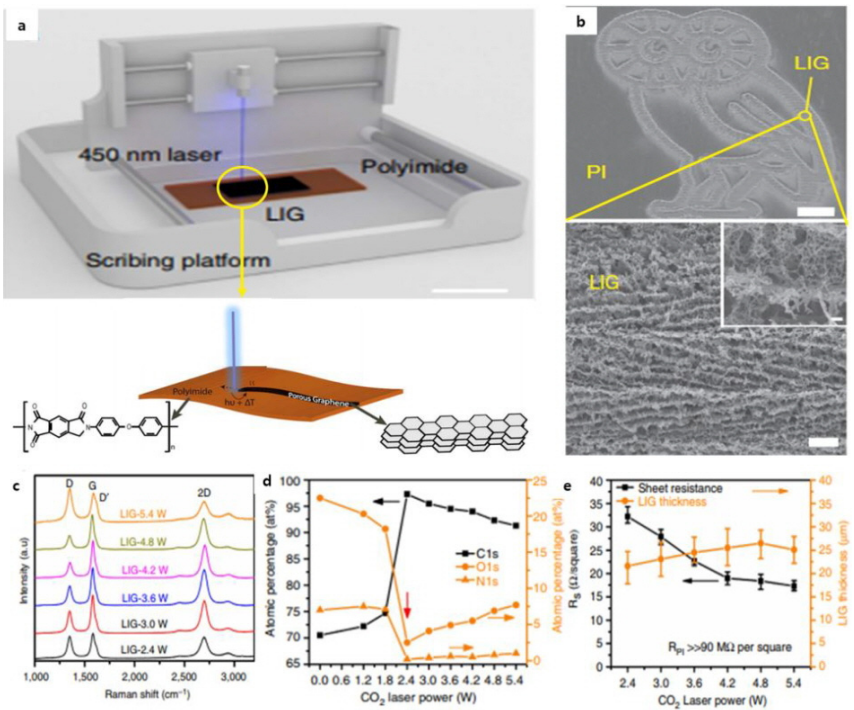

Figure 4. (Color online) (a) Schematic of LIG synthesis on a polyimide (PI) substrate. (Reprinted with permission from [32] (L.-Q. Tao et al., Nat. Comm. 8, 14579 (2017)), ( 2019, Nature Publishing Group). (b) SEM image of LIG. The enlarged image of the circular portion represents the front side of the LIG; the inset shows the back side. (c) Raman spectra of LIG films obtained under different laser powers. (d) Atomic percentage of carbon, oxygen, and nitrogen from XP spectroscopy. (e) Dependence of sheet resistance $\left(R_{\mathrm{s}}\right)$ and LIG thickness on laser power. (Reprinted with permission from [15] (J. Lin et al., Nat. Commun. 5, 5714 (2014)), @ 2019, Nature Publishing Group).

polymers that can be used as raw materials include polyimide (PI), phenolic resin, polyethyleneimine, and lignocellulose [15,33-35].

Notably, when LIG is synthesized on a PI film, a 3D porous structure can be easily formed by using the gas produced during carbonization. The resultant LIG material has an increased specific surface area in comparison to graphene with a planar structure, which improves the capacitance and performance. SEM images of LIG [Fig. 4(b)] reveal a well-defined pattern of porous graphene with clear edges. Similarly to LRG, the characteristics of LIG can be modulated by changing the laser power. Figure 4(c) shows the Raman spectra depending on the laser power. The crystallite size, which can be calculated from the intensity ratio of the $G / D$ bands, increases with increasing laser power. However, when the laser power is higher than the critical power, the crystallite size decreases due to deterioration. Figure 4(d) shows the XP spectra of LIG formed from a PI film as a function of the irradiation power. When the wavelength was $10.6 \mu \mathrm{m}$, graphitization began at $2.4 \mathrm{~W}$. The laser power also affects the thickness and sheet resistance of the LIG [Fig. 4(e)]. By observing the correlation between sheet resistance and laser power, it is clear that excessive power induces damage or oxidation of the graphene instead of crystallization, thereby decreasing the sheet resistance [15].

\section{Functionalization}

Multifunctional graphene can be fabricated by doping LIG materials with metal or metal oxide NPs, as well as by controlling the macro- and/or microstructure of the material. Figure 5(a) shows the fabrication process of metal NP-embedded LIG. By mixing a poly (acrylic acid) solution with a metal precursor, a metal NP/LIG composite can be directly created through a laser-based photothermal process [36]. Transmission electron microscopy (TEM) images confirm 


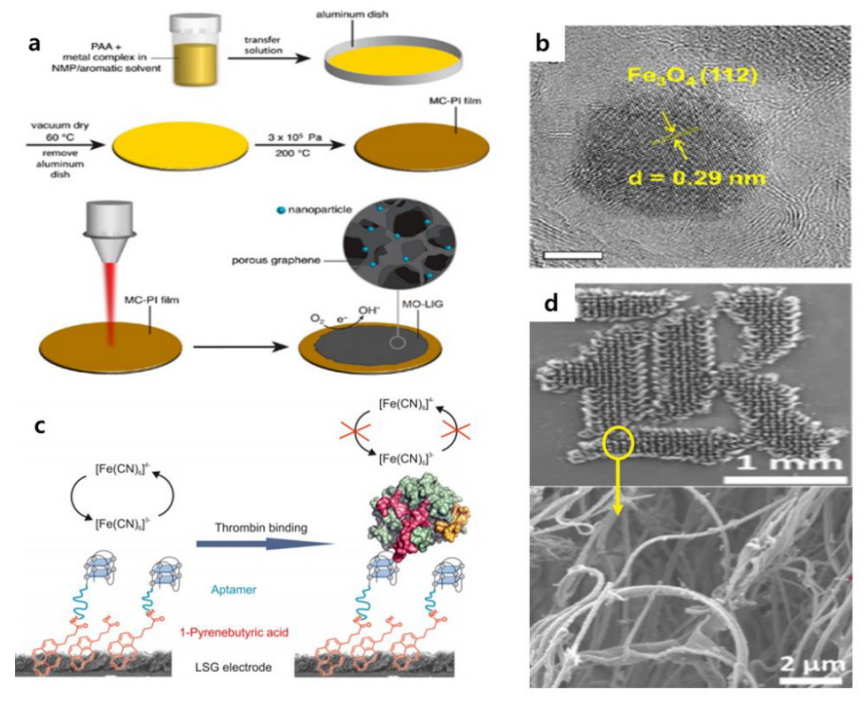

Figure 5. (Color online) (a) Schematic of MO-LIG formation from MC-PI film. (b) TEM image of metal oxide and LIG composite. (Reprinted with permission from [36] (R. Ye et al., ACS Nano 9, 9244 (2015)), (C 2019, American Chemical Society). (c) Schematic of 1-pyrenebutyric acid (PBA) functionalization on LIG. (Reprinted with permission from [37] (C. Fenzl et al., ACS Sens. 2, 616 (2017)), ( ) 2019, American Chemical Society). (d) SEM image of LIG fiber (top) and enlarged view (bottom). (Reprinted with permission from [38] (L. X. Duy et al., Carbon 126, 472 (2018)), ( ) 2019, Elsevier).

that the LIG contains a catalytic metal of spherical $\mathrm{Fe}_{3} \mathrm{O}_{4}$ or $\mathrm{Co}_{3} \mathrm{O}_{4}$ with a diameter of $50 \mathrm{~nm}$; nevertheless, the crystalline structure is maintained [Fig. 5(b)]. It has been reported that doping with metal NPs can improve the performance of LIG-based oxygen reduction reaction electrodes through electrocatalysis [36].

The functionalization of LIG has been shown to facilitate biorecognition. Fenzl et al. [37] introduced a 1-pyrenebutyric acid (PBA) modification method for LIG via hydrophobic interactions. Owing to the high electron transfer rate as well as the excellent peak current response, the PBA-functionalized LIG electrode could effectively detect small amounts of thrombin [Fig. 5(c)]. Duy et al. [38] presented LIGs with diverse macroscopic structures by controlling the laser power, pulses per inch, and process gas. As shown in Fig. 5(d), fiber-type LIG was first synthesized on a PI substrate. It is worth noting that LRG cannot be made with a fiber structure, owing to the limited amount of deformation that can occur during the laser ablation of GO. In addition, it has been reported that LIG fibers have a larger area of active sites to store charges in comparison to conventional porous LIG or LRG [38].

\section{Sensing applications of LIG}

\section{1) Mechanical sensors \\ Strain sensors}

Luo et al. [39] investigated the use of LIG synthesized from PI films for highly sensitive piezoresistive strain sensors. As the electrical conductivity of graphene and the number of contacts within the microstructure in LIG change under strain-induced structural deformation, LIG-based strain sensors have been shown to have a high gauge factor ( $G F=\triangle R \times L / \triangle L \times R$, where $L$ is the length of the sensor and $R$ is the resistance) of above 110; this implies that even tiny
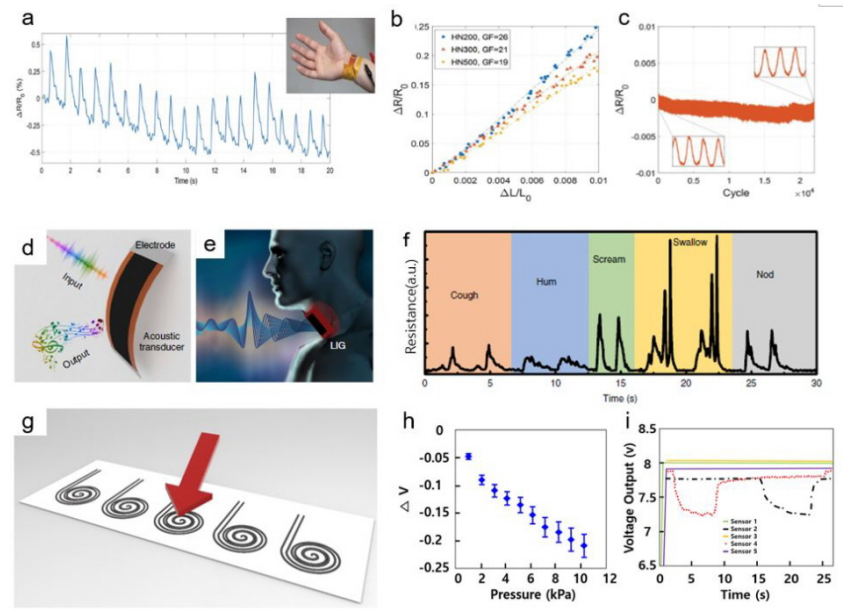

Figure 6. (Color online) (a) Pulse detection by wrapping a wrist with a LIG-based with strain gauge. (b) Forward and backward strain measurements of a LIG-based strain sensor with different substrate thicknesses, exhibiting a linear response. (c) Endurance test of the sensor in (b) $\left(>10^{4}\right.$ cycles). (Reprinted with permission from [42] (A. F. Carvalho et al., Adv. Funct. Mater. 28, 1805271 (2018)), (C 2019, WileyVCH). (d) Schematic showing sound detection and emission using an LIG device. (e) Schematic showing an LIG artificial throat by attaching the device in (e) to a neck. (f) Resistance change of LIG device in (e) upon different sounds and vibrations. (Reprinted with permission from [32] (L.Q. Tao et al., Nat. Comm. 8, 14579 (2017)), (C) 2019, Nature Publishing Group). (g) Schematic of LIG-based pressure sensor array (the arrow represents the application of pressure). (h) Change in output voltage of the sensor in (g) with pressure. (i) Voltage change when two of the five sensors in $(\mathrm{g})$ are sequentially pressed.

movements can effectively be recognized [39]. The thickness of the substrate is as important as that of the active layer, as it influences the extent of force-driven transduction $[40,41]$. Therefore, controlling the thickness of the PI film is crucial for optimizing the performance of these strain sensors. However, the typically used $\mathrm{CO}_{2}$ laser has a wavelength of $10.6 \mu \mathrm{m}$ and high transmissivity on a PI substrate, resulting in deep penetration. In this reason, minimization of the substrate thickness is considerably limited. Nevertheless, thinner and more sensitive sensors can be fabricated by using an ultraviolet (UV) laser [Fig. 6(a)]. The demonstrated gauge sensor with thinner PI film showed five-times enhanced sensitivity and a much better signal-tonoise ratio compared with previously reported LIG sensor. Because the UV laser $(\lambda=355 \mathrm{~nm})$ has a high absorption rate, it can be used to produce thinner and narrower devices. Figure 6(b) shows how the resistance of LIG-based strain sensors with different substrate thicknesses changes with tensile stress. A linear relationship was observed based between strain and resistance for all the sensors; however, the thinner the substrate, the higher the sensitivity. The sensor was subjected to endurance testing by cyclic loading and unloading [Fig. 6(c)]; even after more than $2 \times 10^{4}$ cycles, the sensor retained its original sensitivity [42].

\section{Sound sensors}

LIG is suitable for both sound-emitting and sound-detecting applications owing to its thermoacoustic properties, high thermal conductivity, and low thermal capacity [32]. Figure 6(d) illustrates the soundwaves emitted from a LIG device, which are generated when an alternating current (AC) is applied to the sensor, because periodic Joule heating in the LIG device induces air expansion. Interestingly, 
the vibration of vocal cords can be monitored by measuring the change in the resistance of the device. Accordingly, sound emission and detection can be performed simultaneously, facilitating applications in artificial throat and sound sensors [Fig 6(e)]. Figure 6(f) shows how the resistance of an artificial LIG throat device changes with different sound types (coughing, humming, screaming, swallowing, and nodding). When the same type of sound was repeated, very similar resistance changes were observed. In the cases of swallowing and nodding, the resistance changes induced by the movements of muscles around the neck could be clearly seen [32].

\section{Pressure sensors}

Kazemzadeh et al. [43] and C. Petridis et al. [44] use a scalable one-step process by selectively reducing the GO coating on a GO-rGO complex structure with a laser and apply on pressure sensor [Fig. 6(g)]. The patterned rGO structure on the GO film exhibited excellent sensitivity for pressure. When vertical pressure was applied to the sensor, the conductivity of the system changed owing to the variation in contact area between the rGO sheets. Consequently, the voltage variation and applied pressure had a linear relationship, as shown in Fig. 6(h). Figure 6(i) shows how the resistance changes when two of the sensors in the array are sequentially pressed and released in $10 \mathrm{~s}$ intervals. As the pressure increases, the output voltage increases [45].

\section{2) Electrochemical sensors \\ Gas sensors}

LIG synthesized from PI has a 3D porous structure and a large surface area compared with conventional LIG. The expanded surface area leads to an extreme increase in the number of adsorption sites for gas molecules. Therefore, it is considered a suitable material for improving the sensitivity for gas sensing. Figures 7 (a) and 7(b) show how the resistance of a LIG-based gas sensor changes based on the interaction with a target gas $\left(\mathrm{NH}_{3}\right.$ and $\mathrm{NO}_{2}$, respectively) [46]. LIG has p-type characteristics in air. When exposed to electron-donating and electron-accepting gases $\left(\mathrm{NH}_{3}\right.$ and $\mathrm{NO}_{2}$, respectively), the resistance across the graphene sample increased and decreased, respectively.

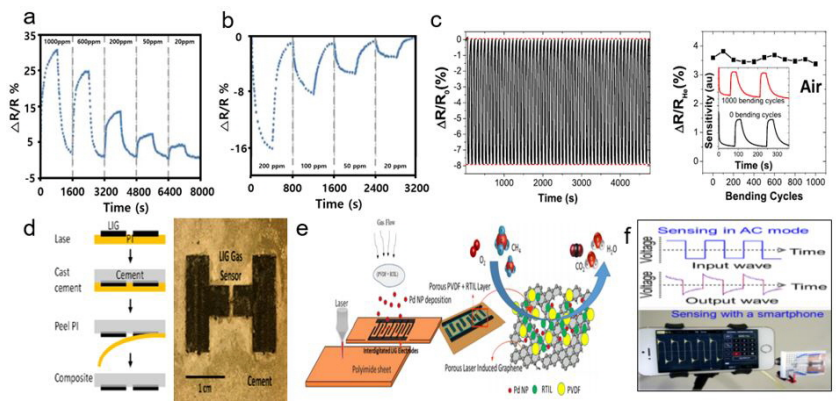

Figure 7. (Color online) (a,b) Response of graphene-based gas sensor for $\mathrm{NH}_{3}$ and $\mathrm{NO}_{2}$, respectively. (c) Bending cycle test of LIG gas sensor (left) and gas sensor test comparison after bending test (right). (d) Fabrication of LIG-based sensor embedded in cement. (Reprinted with permission from [47] (M. G. Stanford et al., ACS Nano 13, 3474 (2019)), @ 2019, American Chemical Society). (e) Schematic of methane sensor fabrication and mechanism using Pd NPs and PVDF/RTIL. (Reprinted with permission from [50] (M. Dosi et al., ACS Appl. Mater. Interfaces 11, 6166 (2019)), (C) 2019, American Chemical Society). (f) Sensing response of LSG humidity sensor (top) and application as portable sensor device (bottom). (Reprinted with permission from [52] (J. Cai et al., ACS Appl. Mater. Interfaces 10, 23987 (2018)), (c) 2019, American Chemical Society).
Nevertheless, in both cases, the sensitivity decreased as the concentration of the gas decreased. When a LIG gas sensor was subjected to bending tests for more than 1000 cycles with a curvature radius of 7 $\mathrm{mm}$, the variation in resistance was uniform and repeatable [Fig. 7(c)]. Therefore, LIG gas sensors simultaneously reflect the excellent electrical properties and mechanical flexibility of graphene, implying that they can be used for bendable and stretchable gas sensor applications.

The porous characteristics of LIG facilitate the formation of functional composites with other materials. Figure $7(\mathrm{~d})$ shows the fabrication process and an optical image of a LIG/cement composite sensor. First, cement slurry is intercalated into the pore structure and anchored with LIG. Subsequently, the PI substrate is removed by thermal treatment, forming a carbon electronic structure embedded in the concrete. The sensor embedded in the composite system is free from corrosion owing to the surrounding environment [47].

Combining LIG gas sensors with a solid-state ionic liquid and metal particles can improve the sensitivity and detection limit. Figure $7(\mathrm{e})$ illustrates a gas sensor fabricated by depositing a mixture of porous polyvinylidene difluoride (PVDF), Pd NPs, and a roomtemperature ionic liquid (RTIL) upon a LIG electrode. The Pd NPs attach to the LIG, increasing its sensitivity to the target gas. In addition, the porous PVDF/RTIL layer increases the porosity of the sensor structure, which creates a larger contact area and flow path, leading to a faster response time. The formation of such composites is of great importance for the development of improved gas sensors [48].

\section{Humidity sensors}

Humidity sensors are widely applied in indoor humidity measurements, weather observations, and industrial applications [49-51]. Cai et al. [52] fabricated a portable humidity sensor by forming a local rGO electrode using partial laser scribing after dispersing GO on a polyethylene terephthalate substrate. The device was very flexible and did not require additional metal electrodes. Figure $7(\mathrm{f})$ illustrates the result of exposing such a humidity sensor with a $\mathrm{rGO} / \mathrm{GO} / \mathrm{rGO}$ structure to moisture. Conventional electrochemical sensors have a low reaction rate because they generally measure the response in the direct current (DC) mode. However, this humidity sensor has a more stable and excellent reactivity in real time, because an $\mathrm{AC}$ measurement system is used. In contrast to the DC mode, an AC mode with a certain period and periodic peak-to-peak voltage enables the response to be checked through the modified output frequency after setting the input frequency, as shown in Fig. 7(f) (top). Figure 7(f) (bottom) shows how the sensor can be formed into a portable humidity sensor system. An oscilloscope cell-phone application is used to confirm that the output wave is produced. Connecting such a sensor to an easily accessible device, such as a cell-phone, demonstrates the applicability of these sensors in a broader sense; i.e., that they can replace conventional large measurement systems and operate in connection with a network [52].

\section{Summary}

In this review, a quick, inexpensive, and simple synthesis method for porous graphene with a $3 \mathrm{D}$ structure is presented, along with the sensor applications of such materials. The facile laser-based thermal annealing approach allows the large-area synthesis of graphene devices with diverse microscale architectures within a few minutes. Notably, 
this method overcomes the drawbacks of conventional graphene-based sensor fabrication, which requires high temperatures, vacuum conditions, and multiple process steps. Laser scribing can be used to readily fabricate electromechanical and electrochemical sensors, including piezoresistive-based strain sensors, pressure sensors, artificial throat and sound sensors, hazardous gas sensors, and humidity sensors. The function of graphene can be optimized by controlling the laser wavelength, working distance, and structure of the source materials. This attractive laser-based method of synthesizing functionalized graphene is emerging as a key method owing to its advantages such as diverse material property control, high processing speed, and low production cost; it is expected to be adopted in the industry beyond the laboratory scale in the near future.

\section{Acknowledgements}

This research was supported by the Korea Institute of Science and Technology (KIST) Institutional Program and Jeong-in Engineering Program.

\section{References}

[1] D. H. Ho, Q. Sun, S. Y. Kim, J. T. Han, D. H. Kim, and J. H. Cho, Adv. Mater. 28, 2601 (2016).

[2] T. Wang, D. Huang, Z. Yang, S. Xu, G. He, X. Li, N. Hu, G. Yin, D. He, and L. Zhang, Nano-Micro Lett. 8, 95 (2016).

[3] A. Star, V. Joshi, S. Skarupo, D. Thomas, and J. C. P. Gabriel, J. Phys. Chem. B 110, 21014 (2006).

[4] Q. Wan, Q. H. Li, Y. J. Chen, T. H. Wang, X. L. He, J. P. Li, and C. L. Lin, Appl. Phys. Lett. 84, 3654 (2004).

[5] A. Peigney, Ch. Laurent, E. Flahaut, R. R. Bacsa, and A. Rousset, Carbon 39, 507 (2001).

[6] S. Stankovich, D. A. Dikin, G. H. B. Dommett, K. M. Kohlhaas, E. J. Zimney, E. A. Stach, R. D. Piner, S. T. Nguyen, and R. S. Ruoff, Nature 442, 282 (2006).

[7] S. Rumyantsev, G. Liu, M. S. Shur, R. A. Potyrailo, and A. A. Balandin, Nano Lett. 12, 2294 (2012).

[8] D. R. Kauffman and A. Star, Angew. Chem. Int. Ed. 47, 6650 (2008).

[9] F. Schedin, A. K. Geim, S. V. Morozov, E. W. Hill, P. Blake, M. I. Katsnelson, and K. S. Novoselov, Nat. Mater. 6, 652 (2007).

[10] C. Yan, J. Wang, W. Kang, M. Cui, X. Wang, C. Y. Foo, K. J. Chee, and P. S. Lee, Adv. Mater. 26, 2022 (2014).

[11] K. S. Novoselov, A. K. Geim, S. V. Morozov, D. Jiang, Y. Zhang, S. V. Dubonos, I. V. Grigorieva, and A. A. Firsov, Science 306, 666 (2004).

[12] X. Li, W. Cai, J. An, S. Kim, J. Nah, D. Yang, R. Piner, A. Velamakanni, I. Jung, E. Tutuc, S. K. Banerjee, L. Colombo, and R. S. Ruoff, Science 324, 1312 (2009).

[13] C. Berger, Z. Song, X. Li, X. Wu, N. Brown, C. Naud, D. Mayou, T. Li, J. Hass, A. N. Marchenkov, E. H. Conrad, P. N. First, and W. A. de Heer, Science 312, 1191 (2006).

[14] Y. Zhang, L. Guo, S. Wei, Y. He, H. Xia, Q. Chen, H. B. Sun, and F. S. Xiao. Nano Today 5, 15 (2010).

[15] J. Lin, Z. Peng, Y. Liu, F. Ruiz-Zepeda, R. Ye, E. L. G. Samuel, M. J. Yacaman, B. I. Yakobson, and J. M. Tour, Nat. Commun. 5, 5714 (2014).

[16] R. Arul, R. N. Oosterbeek, J. Robertson, G. Xu, J. Jin, and M. C.
Simpson, Carbon 99, 423 (2016).

[17] M. Qian, Y. S. Zhou, Y. Gao, J. B. Park, T. Feng, S. M. Huang, Z. Sun, L. Jiang, and Y. F. Lu, Appl. Phys. Lett. 98, 173108 (2011).

[18] L. Huang, Y. Liu, L. C. Ji, Y. Q. Xie, T. Wang, and W. Z. Shi, Carbon 49, 2431 (2011)

[19] W. Gao, N. Singh, L. Song, Z. Liu, A. L. M. Reddy, L. Ci, R. Vajtai, Q. Zhang, B. Wei, and P. M. Ajayan, Nat. Nanotechnol. 6, 496 (2011).

[20] M. F. El-Kady, V. Strong, S. Dubin, and R. B. Kaner, Science 355, 1326 (2012).

[21] X. Ye, J. Long, Z. Lin, H. Zhang, H. Zhu, and M. Zhong, Carbon 68, 784 (2014).

[22] V. Strong, S. Dubin, M. F. El-Kady, A. Lech, Y. Wang, B. H. Weiller, and R. B. Kane, ACS Nano 6, 1395 (2012).

[23] H. Tian, Y. Shu, Y. L. Cui, W. T. Mi, Y. Yang, D. Xie, and T. L. Ren, Nanoscale 6, 699 (2014).

[24] H. Tian, Y. Yang, D. Xie, Y. L. Cui, W. T. Mi, Y. Zhang, and T. L. Ren, Sci. Rep. 4, 3598 (2014).

[25] V. A. Smirnov, A. A. Arbuzov, Yu. M. Shul'ga, S. A. Baskakov, V. M. Martynenko, V. E. Muradyan, and E. I. Kresova, High Energy Chem. 45, 57 (2011).

[26] Z. Wan, E. W. Streed, M. Lobino, S. Wang, R. T. Sang, I. S. Cole, D. V. Thiel, and Q. Li, Adv. Mater. Technol. 3, 1700315 (2018).

[27] L. Guo, H. B. Jiang, R. Q. Shao, Y. L. Zhang, S. Y. Xie, J. N. Wang, X. B. Li, F. Jiang, Q. D. Chen, T. Zhang, and H. B. Sun, Carbon 50, 1667 (2012).

[28] Y. Chen, J. Xu, Y. Yang, Y. Zhao, W. Yang, X. He, S. Li, and C. Jia, J. Mater. Sci.: Mater. Electron. 27, 2564 (2016).

[29] W. H. Lee, J. W. Suk, H. Chou, J. Lee, Y. Hao, Y. Wu, R. Piner, D. Akinwande, K. S. Kim, and R. S. Ruoff, Nano Lett. 12, 2374 (2012).

[30] H. Cheng, J. Liu, Y. Zhao, C. Hu, Z. Zhang, N. Chen, L. Jiang, and L. Qu, Angew. Chem. Int. Ed. 52, 10482 (2013).

[31] H. Cheng, M. Ye, F. Zhao, C. Hu, Y. Zhao, Y. Liang, N. Chen, S. Chen, L. Jiang, and L. Qu, Adv. Mater. 28, 3305 (2016).

[32] L. Q. Tao, H. Tian, Y. Liu, Z. Y. Ju, Y. Pang, Y. Q. Chen, D. Y. Wang, X. G. Tian, J. C. Yan, N. Q. Deng, Y. Yang, and T. L. Ren, Nat. Commun. 8, 14579 (2017).

[33] Z. Zhang, M. Song, J. Hao, K. Wu, C. Li, and C. Hu, Carbon 127, 287 (2018).

[34] Y. Chyan, R. Ye, Y. Li, S. P. Singh, C. J. Arnusch, and J. M. Tour, ACS Nano 12, 2176 (2018).

[35] R. Ye, Y. Chyan, J. Zhang, Y. Li, X. Han, C. Kittrell, and J. M. Tour, Adv. Mater. 29, 1702211 (2017).

[36] R. Ye, Z. Peng, T. Wang, Y. Xu, J. Zhang, Y. Li, L. G. Nilewski, J. Lin, and J. M. Tour, ACS Nano 9, 9244 (2015).

[37] C. Fenzl, P. Nayak, T. Hirsch, O. S. Wolfbeis, H. N. Alshareef, and A. J. Baeumner, ACS Sens. 2, 616 (2017).

[38] L. X. Duy, Z. Peng, Y. Li, J. Zhang, Y. Ji, and J. M. Tour, Carbon 126,472 (2018).

[39] S. Luo, P. T. Hoang, and T. Liu, Carbon 96, 522 (2016).

[40] R. Rahimi, M. Ochoa, W. Yu, and B. Ziaie, ACS Appl. Mater. Interfaces 7, 4463 (2015).

[41] D. X. Luong, A. K. Subramanian, G. A. Lopez Silva, J. Yoon, S. Cofer, K. Yang, P. S. Owuor, T. Wang, Z. Wang, J. Lou, P. M. Ajayan, and J. M. Tour, Adv. Mater. 30, 1707416 (2018).

[42] A. F. Carvalho, A. J. S. Fernandes, C. Leitão, J. Deuermeier, A. C. Marques, R. Martins, E. Fortunato, and F. M. Costa, Adv. Funct. 
Mater. 28, 1805271 (2018).

[43] R. Kazemzadeh, K. Andersen, L. Motha, and W. S. Kim, IEEE Electron Device Lett. 36, 180 (2014).

[44] C. Petridis, Y. H. Lin, K. Savva, G. Eda, E. Kymakis, T. D. Anthopoulos, and E. Stratakis, Appl. Phys. Lett. 102, 093115 (2013).

[45] H. B. Yao, J. Ge, C. F. Wang, X. Wang, W. Hu, Z. J. Zheng, Y. Ni, and S. H. Yu, Adv. Mater. 25, 6692 (2013).

[46] L. Kong, A. Enders, T. S. Rahman, and P. A. Dowben, J. Phys.: Condens. Matter 26, 443001 (2014).

[47] M. G. Stanford, K. Yang, Y. Chyan, C. Kittrell, and J. M. Tour,
ACS Nano 13, 3474 (2019).

[48] M. Dosi, I. Lau, Y. Zhuang, D. S. A. Simakov, M. W. Fowler, and M. A. Pope, ACS Appl. Mater. Interfaces 11, 6166 (2019).

[49] H. Farahani, R. Wagiran, and M. N. Hamidon, Sensors 14, 7881 (2014).

[50] T. A. Blank, L. P. Eksperiandova, and K. N. Belikov, Sens. Actuators B 228, 416 (2016).

[51] Z. Chen and C. Lu, Sens. Lett. 3, 274 (2005).

[52] J. Cai, C. Lv, E. Aoyagi, S. Ogawa, and A. Watanabe, ACS Appl. Mater. Interfaces 10, 23987 (2018). 